\title{
Application of mathematical methods of engineering to transient analysis of r-l-c series circuit with sinusoidal voltage
}

\author{
Anuradha S Deshpande \\ Associate professor, Department of Elect. Engineering, Faculty of Technology \& Engineering, \\ Maharaja Sayajirao University of Baroda, Vadodara 390001 \\ E-mail: anuradhasdeshpande62@gmail.com
}

Copyright (c) 2014 Anuradha S Deshpande. This is an open access article distributed under the Creative Commons Attribution License, which permits unrestricted use, distribution, and reproduction in any medium, provided the original work is properly cited.

\begin{abstract}
The paper has made an attempt to obtain transient response of an AC series circuit by mathematical methods of engineering namely classical method and Laplace transform method. The equations developed are differential equations of second order. The solution is obtained by classical method with initialization condition and Laplace transform method. A comparative study is done in analyzing the transient response of an $\mathrm{R}-\mathrm{L}-\mathrm{C}$ series circuit connected to sinusoidal source of voltage by the two methods. The derived equations of transient response by two methods are similar although Laplace transform method is better tool with respect to initialization conditions in a circuit. Proposed work has established similarity of transient response of all the three conditions of under and over damped, as well as critically damped case by two different methods.
\end{abstract}

Keywords: Classical Method, Differential Equation, Initialization, Laplace Transforms Transient Response.

\section{Introduction}

Transient response of An AC series circuit is obtained by traditional methods like classical method as well as more generalized methods as Laplace transform method. In case of R-L-C series circuit connected to sinusoidal source of voltage $\mathrm{v}=\mathrm{V}_{\mathrm{m}} \sin \omega \mathrm{t}$, a non-homogeneous differential equation of second order is formed which is solved.

For a differential equation, the complete solution consists of two parts; the complementary function and the particular solution. The complementary function dies out after short interval and is referred to as the transient response or source free response. The particular solution is the steady state response, or the forced response. The first step in finding the complete solution of a circuit is to form a differential equation for the circuit. Differential equation can be solved by several methods to find out the complete solution.

The transient response of R-L-C AC series circuit is important due to the fact that actual transmission line undergoes similar phenomena. The current through the transmission line is affected due to application of voltage and also due to withdrawal of voltage or case of sudden switching. The performance of the transmission line is due to steady state condition and transient condition. Therefore need is to evaluate the characteristic equation of the current due to Forced and Natural response. Also transient response is classified in to over damped, under damped and critically damped case. Thus paper attempts to analyse and establish similarity with 3 phase transmission line performance.

\subsection{Problem formulation}

Fig 1 shows a circuit consisting of resistance, inductance and capacitance connected in series to sinusoidal source of voltage. Inductance and capacitance are in uncharged state initially.

The current equation consists of two parts namely one due to application of voltage source called as forced response. Fig 2 indicates flow of current. 


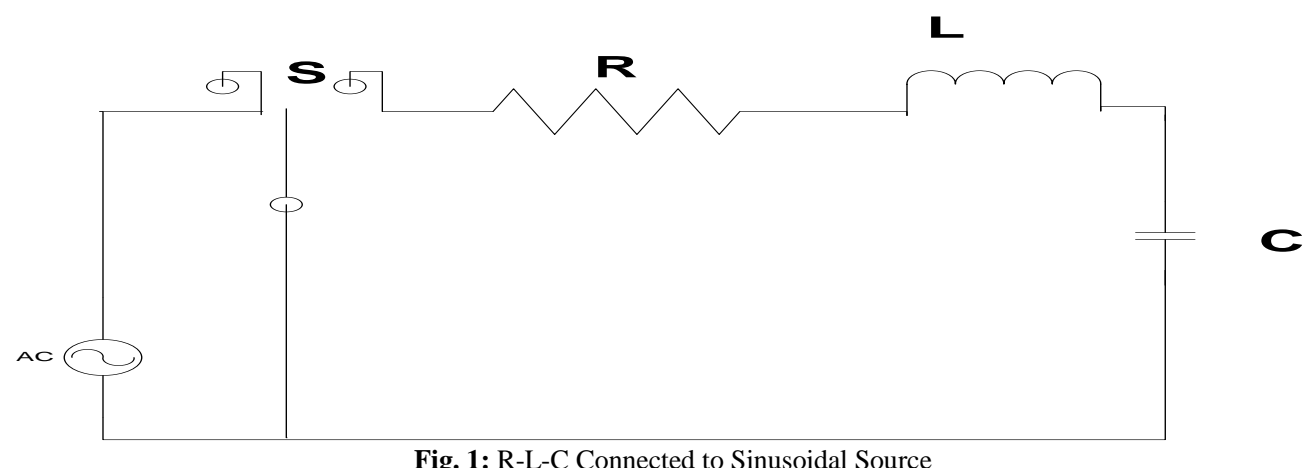

Fig. 1: R-L-C Connected to Sinusoidal Source

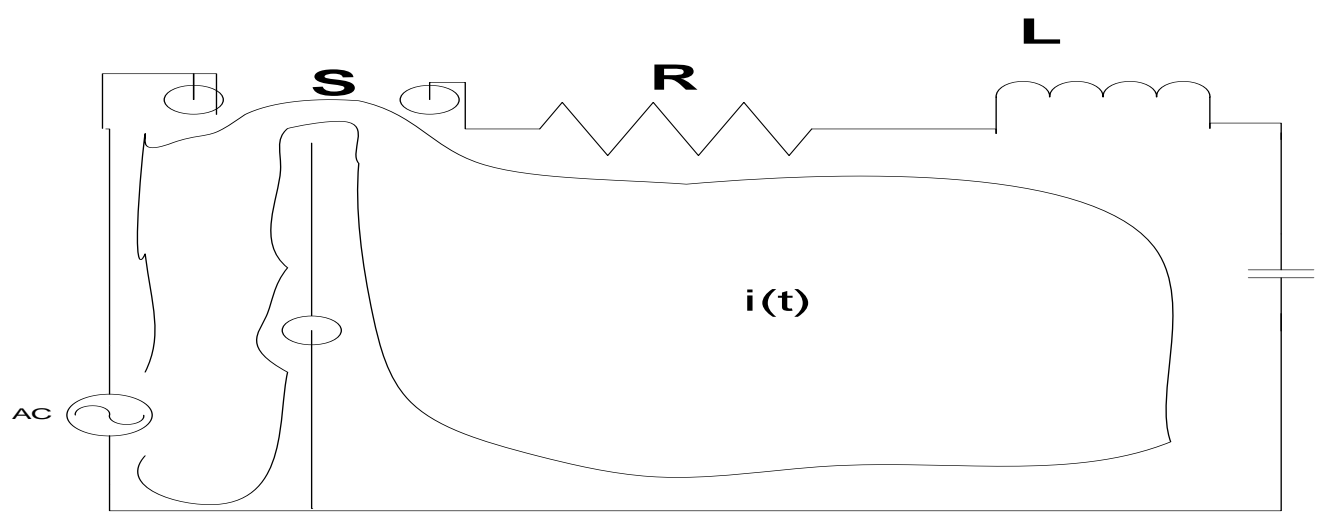

Fig. 2: Current Due to Forced Response

The other part of the current is due to withdrawal of the source called as Natural or transient response. Fig 3 indicates flow of current.

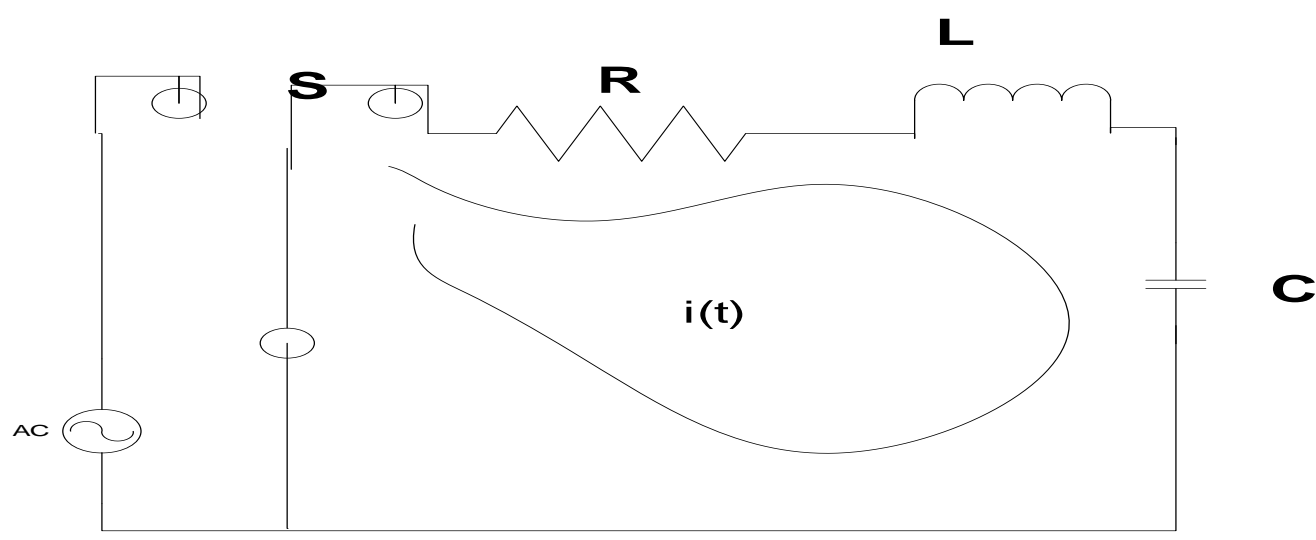

Fig. 3: Current Due to Natural Response

\subsection{Solution methodology}

The solution of above problem is obtained by Classical method and also by Laplace transform method.

\subsubsection{Classical method}

Consider the circuit consisting of resistance, inductance and capacitance connected in series to sinusoidal source of voltage.

Current circulates through the circuit when switch $S$ is closed and application of sinusoidal voltage, Vsin $(\omega t+$ $\theta$,where Vis the amplitude of the wave and $\theta$ is the phase angle.

Switch $\mathrm{S}$ is closed at $\mathrm{t}=0$. 


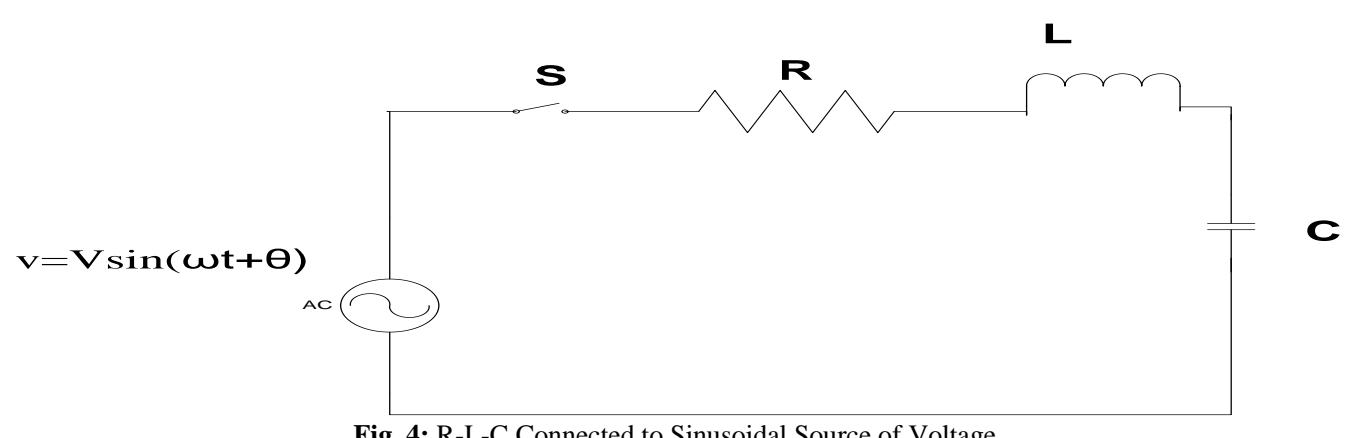

Fig. 4: R-L-C Connected to Sinusoidal Source of Voltage

Applying KVL to the circuit results in the following differential equation.

$\operatorname{Vsin}(\omega t+\theta)=i R+\frac{1}{C} \int i d t+L \frac{d i}{d t}$

Differentiating above equation (1), we get

$\underline{\mathrm{V} \omega \cos (\omega \mathrm{t}+\theta)}=\mathrm{L} \frac{\mathrm{d}^{2} \mathrm{i}}{\mathrm{dt}^{2}}+\frac{1}{\mathrm{C}} \mathrm{i}+\mathrm{iR}$ or

$\frac{\mathrm{V} \omega \cos (\omega \mathrm{t}+\theta)}{\mathrm{L}}=\frac{\mathrm{d}^{2} \mathrm{i}}{\mathrm{dt}^{2}}+\frac{\mathrm{R}}{\mathrm{L}} \frac{\mathrm{di}}{\mathrm{dt}}+\frac{\mathrm{i}}{\mathrm{LC}}$

$D^{2}+\frac{R}{L} \frac{d i}{d t}+\frac{i}{L C}=\frac{V \omega \cos (\omega t+\theta)}{L}$

Solution of above equation is $i(t)=i_{p}+i_{h}$ where $i_{p}$ is the particular solution and $i_{h}$ is the complementary solution. $i_{p}$ is obtained by solution of non-homogeneous linear differential equation

$\mathrm{D}^{2}+\frac{\mathrm{R}}{\mathrm{L}} \frac{\mathrm{di}}{\mathrm{dt}}+\frac{\mathrm{i}}{\mathrm{LC}}=\frac{\mathrm{V} \omega \cos (\omega \mathrm{t}+\theta)}{\mathrm{L}}$

And $i_{c}$ is obtained as homogeneous linear differential equation.

$\mathrm{D}^{2}+\frac{\mathrm{R}}{\mathrm{L}} \frac{\mathrm{di}}{\mathrm{dt}}+\frac{\mathrm{i}}{\mathrm{LC}}=0$

Complementary solution of eq (2) is $i_{C}=c e^{\frac{-t}{R C}}$ where value of c can be obtained by applying initial conditions.

For obtaining particular solution, the current solution is estimated as

$\mathrm{i}_{\mathrm{p}}=\mathrm{A} \cos (\omega \mathrm{t}+\theta)+\mathrm{B} \sin (\omega \mathrm{t}+\theta)$

Differentiating eq (6) we get,

$\mathrm{i}_{\mathrm{p}}{ }^{\prime}=-\mathrm{A} \omega \sin (\omega \mathrm{t}+\theta)+\mathrm{B} \omega \cos (\omega \mathrm{t}+\theta)$

$\mathrm{i}_{\mathrm{p}}^{\prime \prime}=-\mathrm{A} \omega^{2} \cos (\omega \mathrm{t}+\theta)-\mathrm{B} \omega^{2} \sin (\omega \mathrm{t}+\theta)$

Substituting the value of $\mathrm{i}_{\mathrm{p}}{ }^{\prime}$ and $\mathrm{i}_{\mathrm{p}}{ }^{\prime \prime}$ in eq (2), we get

$-\mathrm{A} \omega^{2} \cos (\omega \mathrm{t}+\theta)-\mathrm{B} \omega^{2} \sin (\omega \mathrm{t}+\theta)+\frac{\mathrm{R}}{\mathrm{L}}(-\mathrm{A} \omega \sin (\omega \mathrm{t}+\theta)+\mathrm{B} \omega \cos (\omega \mathrm{t}+\theta))+\frac{1[\mathrm{~A} \cos (\omega \mathrm{t}+\theta)+\mathrm{B} \sin (\omega \mathrm{t}+\theta)]}{\mathrm{LC}}=$ $\underline{\mathrm{V} \omega \cos (\omega \mathrm{t}+\theta)}$

Collecting and equating real and imaginary components of (9) on both sides, we get equation

$\mathrm{A}=\frac{\mathrm{V} \omega}{\mathrm{L}} \times \frac{\omega^{2}-\frac{1}{\mathrm{LC}}}{\left(\frac{\omega \mathrm{R}}{\mathrm{L}}\right)^{2}+\left(\omega^{2}-\frac{1}{\mathrm{LC}}\right)^{2}}$

and

$\mathrm{B}=-\frac{\mathrm{V} \omega^{2} \mathrm{R}}{\mathrm{L}^{2}} \times \frac{1}{\left(\frac{\omega \mathrm{R}}{\mathrm{L}}\right)^{2}+\left(\omega^{2}-\frac{1}{\mathrm{LC}}\right)^{2}}$

Substituting value of $\mathrm{A}$ and $\mathrm{B}$ in equation (6) results in to

$\mathrm{i}_{\mathrm{p}}=\frac{\mathrm{V} \omega}{\mathrm{L}} \times \frac{\omega^{2}-\frac{1}{\mathrm{LC}}}{\left(\frac{\omega \mathrm{R}}{\mathrm{L}}\right)^{2}+\left(\omega^{2}-\frac{1}{\mathrm{LC}}\right)^{2}} \cos (\omega \mathrm{t}+\theta)-\frac{\mathrm{V} \omega^{2} \mathrm{R}}{\mathrm{L}^{2}} \times \frac{1}{\left(\frac{\omega \mathrm{R}}{\mathrm{L}}\right)^{2}+\left(\omega^{2}-\frac{1}{\mathrm{LC}}\right)^{2}} \sin (\omega \mathrm{t}+\theta)$

Assuming Mcos

$\varphi=\frac{\mathrm{V} \omega^{2} \mathrm{R}}{\mathrm{L}^{2}} \times \frac{1}{\left(\frac{\omega \mathrm{R}}{\mathrm{L}}\right)^{2}+\left(\omega^{2}-\frac{1}{\mathrm{LC}}\right)^{2}}$

And

$\operatorname{Msin} \varphi=\frac{\mathrm{V} \omega}{\mathrm{L}} \times \frac{\omega^{2}-\frac{1}{\mathrm{LC}}}{\left(\frac{\omega \mathrm{R}}{\mathrm{L}}\right)^{2}+\left(\omega^{2}-\frac{1}{\mathrm{LC}}\right)^{2}} \frac{\mathrm{V} \omega \mathrm{C}}{1+\omega^{2} \mathrm{R}^{2} \mathrm{C}^{2}}$

Dividing equation(14) by(13), we get

$\tan \varphi=\left(\frac{\omega \mathrm{L}-\frac{1}{\omega \mathrm{C}}}{\mathrm{R}}\right)$ or $\varphi=\tan ^{-1}\left(\frac{\omega \mathrm{L}-\frac{1}{\omega \mathrm{C}}}{\mathrm{R}}\right)=\frac{\mathrm{V}}{\text { and } \mathrm{M}}$

With above assumptions of $\operatorname{Mcos} \varphi$ and $\operatorname{Msin} \varphi$ Equation (12) reduces to

$\mathrm{i}_{\mathrm{p}}=\operatorname{Msin} \varphi \cos (\omega \mathrm{t}+\theta)-\operatorname{Mcos} \varphi \sin (\omega \mathrm{t}+\theta)$ 
$\therefore \mathrm{i}_{\mathrm{p}}=\mathrm{M} \sin (\omega \mathrm{t}+\theta-\varphi)$.

Substituting the value of $\mathrm{M}$ and $\varphi$, resulting equation of

$\mathrm{i}_{\mathrm{p}}=\frac{\mathrm{v}}{\sqrt{\mathrm{R}^{2}+\left(\omega \mathrm{L}-\frac{1}{\omega \mathrm{C}}\right)^{2}}} \sin \left(\omega \mathrm{t}+\theta-\tan ^{-1}\left(\frac{\omega \mathrm{L}-\frac{1}{\omega \mathrm{C}}}{\mathrm{R}}\right)\right)$

For obtaining value of complementary function the characteristic equation is $D^{2}+\frac{R}{L} \frac{d i}{d t}+\frac{i}{L C}=0$.

The roots of the characteristic equation are (i) $\mathrm{D}_{1}=\mathrm{k}_{1}+\mathrm{K}_{2}$ and $\mathrm{D}_{2}=\mathrm{k}_{1}-\mathrm{k}_{2}$. Case for over damped state

The resulting current equation is

$\mathrm{i}(\mathrm{t})=\mathrm{i}_{\mathrm{p}}+\mathrm{i}_{\mathrm{c}}=\frac{\mathrm{v}}{\sqrt{\mathrm{R}^{2}+\left(\omega \mathrm{L}-\frac{1}{\omega \mathrm{C}}\right)^{2}}} \sin \left(\omega \mathrm{t}+\theta-\tan ^{-1}\left(\frac{\omega \mathrm{L}-\frac{1}{\omega \mathrm{C}}}{\mathrm{R}}\right)\right)+\left[\mathrm{D}-\left(\mathrm{k}_{1}+\mathrm{k}_{2}\right)\right]\left[\mathrm{D}-\left(\mathrm{k}_{1}-\mathrm{k}_{2}\right) \mathrm{i}\right]$
$\mathrm{i}(\mathrm{t})=\mathrm{i}_{\mathrm{p}}+\mathrm{i}_{\mathrm{c}}=\frac{\mathrm{V}}{\sqrt{\mathrm{R}^{2}+\left(\omega \mathrm{L}-\frac{1}{\omega \mathrm{C}}\right)^{2}}} \sin \left(\omega \mathrm{t}+\theta-\tan ^{-1}\left(\frac{\omega \mathrm{L}-\frac{1}{\omega \mathrm{C}}}{\mathrm{R}}\right)\right)+\left[\mathrm{c}_{1} \mathrm{e}^{\left(\mathrm{k}_{1}+\mathrm{k}_{2}\right) \mathrm{t}}-\mathrm{c}_{2} \mathrm{e}^{\left(\mathrm{k}_{1}-\mathrm{k}_{2}\right) \mathrm{t}}\right]$

(ii) $\mathrm{D}_{1}=\mathrm{k}_{1}+\mathrm{jk} \mathrm{k}_{2}$ and $\mathrm{D}_{2}=\mathrm{k}_{1}-\mathrm{jk}$. Case for under damped state.

$\mathrm{i}(\mathrm{t})=\mathrm{i}_{\mathrm{p}}+\mathrm{i}_{\mathrm{c}}=\frac{\mathrm{v}}{\sqrt{\mathrm{R}^{2}+\left(\omega \mathrm{L}-\frac{1}{\omega \mathrm{C}}\right)^{2}}} \sin \left(\omega \mathrm{t}+\theta-\tan ^{-1}\left(\frac{\omega \mathrm{L}-\frac{1}{\omega \mathrm{C}}}{\mathrm{R}}\right)\right)+\left[\mathrm{D}-\left(\mathrm{k}_{1}+\mathrm{jk} \mathrm{k}_{2}\right)\right]\left[\mathrm{D}-\left(\mathrm{k}_{1}-\mathrm{jk} \mathrm{k}_{2}\right) \mathrm{i}\right]$

$\mathrm{i}(\mathrm{t})=\mathrm{i}_{\mathrm{p}}+\mathrm{i}_{\mathrm{c}}=\frac{\mathrm{v}}{\sqrt{\mathrm{R}^{2}+\left(\omega \mathrm{L}-\frac{1}{\omega \mathrm{C}}\right)^{2}}} \sin \left(\omega \mathrm{t}+\theta-\tan ^{-1}\left(\frac{\omega \mathrm{L}-\frac{1}{\omega \mathrm{C}}}{\mathrm{R}}\right)\right)+\mathrm{e}^{\mathrm{k}_{1} \mathrm{t}}\left[\mathrm{c}_{1} \cos _{2} \mathrm{t}+\mathrm{c}_{2} \operatorname{sink}_{2} \mathrm{t}\right]$

(iii) $\mathrm{D}_{1}=\mathrm{k}_{1}$ and $\mathrm{D}_{2}=\mathrm{k}_{1}$

$i(t)=i_{p}+i_{c}=\frac{v}{\sqrt{R^{2}+\left(\omega L-\frac{1}{\omega C}\right)^{2}}} \sin \left(\omega t+\theta-\tan ^{-1}\left(\frac{\omega L-\frac{1}{\omega C}}{R}\right)\right)+\left[D-\left(k_{1}\right)\right]\left[D-\left(k_{1}\right) i\right]$
$i(t)=i_{p}+i_{c}=\frac{V}{\sqrt{R^{2}+\left(\omega L-\frac{1}{\omega C}\right)^{2}}} \sin \left(\omega t+\theta-\tan ^{-1}\left(\frac{\omega L-\frac{1}{\omega C}}{R}\right)\right)+e^{k_{1} t}\left[c_{1}+c_{2}\right]$

\subsubsection{Laplace transform method}

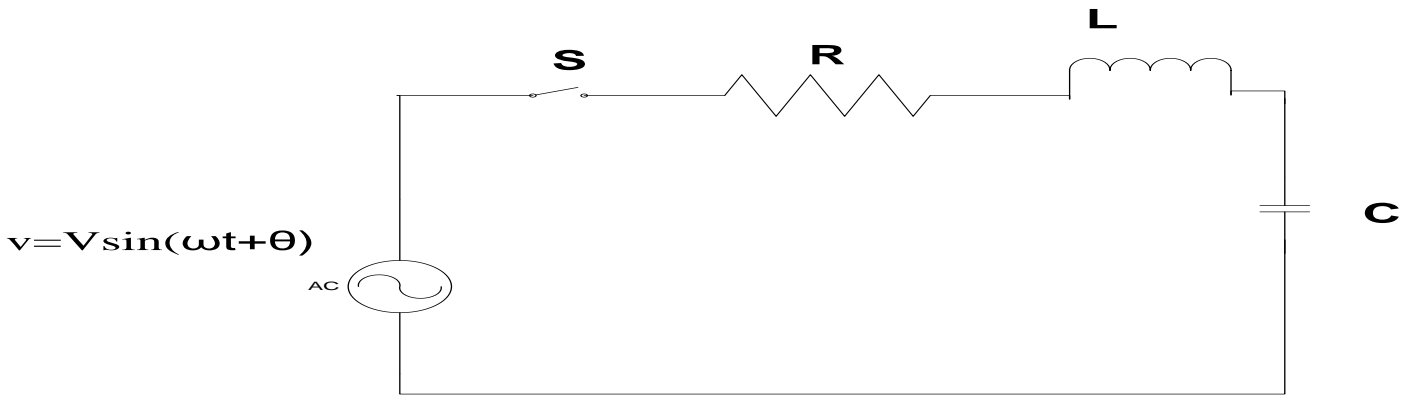

Fig. 5: R-L-C Connected to Sinusoidal Source of Voltage

Consider the circuit consisting of resistance, inductance and capacitor connected in series to sinusoidal source of voltage.

Current circulates through the circuit when switch $\mathrm{S}$ is closed and application of sinusoidal

Voltage $\operatorname{Vsin}(\omega t+\theta)$, where Vis the amplitude of the wave and $\theta$ is the phase angle.

Switch $S$ is closed at $\mathrm{t}=0$.

Applying KVL to the circuit results in the following differential equation.

$\operatorname{Vsin}(\omega \mathrm{t}+\theta)=\mathrm{iR}+\mathrm{L} \frac{\mathrm{di}}{\mathrm{dt}}+\frac{1 \int \mathrm{idt}}{\mathrm{C}}$

Taking Laplace transform of above equation,
$\mathrm{I}(\mathrm{s})\left[\mathrm{Ls}+\mathrm{R}+\frac{1}{\mathrm{Cs}}\right]=\frac{\mathrm{V}(\sin \theta+\omega \cos \theta)}{\mathrm{s}^{2}+\omega^{2}}$
$\mathrm{I}(\mathrm{s})\left[\mathrm{s}^{2}+\frac{\mathrm{R}}{\mathrm{L}} \mathrm{s}+\frac{1}{\mathrm{LC}}=\frac{\mathrm{Vs}}{\mathrm{L}} \frac{(\mathrm{s} \sin \theta+\omega \cos \theta)}{\mathrm{s}^{2}+\omega^{2}}\right]$
$\mathrm{I}(\mathrm{s})=\frac{\mathrm{Vs}}{\mathrm{L}} \frac{(\mathrm{s} \sin \theta+\omega \cos \theta)}{\left(\mathrm{s}^{2}+\omega^{2}\right)\left(\mathrm{s}^{2}+\frac{\mathrm{R}}{\mathrm{L}} \mathrm{s}+\frac{1}{\mathrm{LC}}\right)}$

Where $s_{1}$ and $s_{2}$ are roots of the characteristic equation? $\left(s^{2}+\frac{R}{L} s+\frac{1}{L C}\right)=0$

$I(s)=\frac{V s}{L} \frac{(s \sin \theta+\omega \cos \theta)}{(s+j \omega)(s-j \omega)\left(s-s_{2}\right)\left(s-s_{1}\right)} S_{1}, S_{2},=\frac{-R}{2 L} \pm \sqrt{\left(\frac{R}{2 L}\right)^{2}-\frac{1}{L C}}$

Expressing RHS of equation (27) we get, 
Let $\frac{V s}{L} \frac{(s \sin \theta+\omega \cos \theta)}{(s+j \omega)(s-j \omega)\left(s-s_{2}\right)\left(s-s_{1}\right)}=\frac{k_{1}}{\left(s-s_{1}\right)}+\frac{k_{2}}{\left(s-s_{2}\right)}+\frac{k_{3}}{(s+j \omega)}+\frac{k_{4}}{s-j \omega}$

Using Laplace method to determine coefficient onRHS,

Multiplying eq (28) on both sides by $\left(s-s_{1}\right)$, and putting $s=s_{1}$ we get

$\frac{\mathrm{V}}{\mathrm{L}} \frac{\mathrm{s}(\mathrm{s} \sin \theta+\omega \cos \theta)\left(\mathrm{s}-\mathrm{s}_{1}\right)}{(\mathrm{s}+\mathrm{j} \omega)(\mathrm{s}-\mathrm{j} \omega)\left(\mathrm{s}-\mathrm{s}_{2}\right)\left(\mathrm{s}-\mathrm{s}_{1}\right)}=\mathrm{k}_{1}+0$.

$\therefore \mathrm{k}_{1}=\frac{\mathrm{V}}{\mathrm{L}} \frac{\mathrm{s}_{1}\left(\mathrm{~s}_{1} \sin \theta+\omega \cos \theta\right)}{\left(\mathrm{s}_{1}+\mathrm{j} \omega\right)\left(\mathrm{s}_{1}-\mathrm{j} \omega\right)\left(\mathrm{s}_{1}-\mathrm{s}_{2}\right)}$

Multiplying eq (28) on both sides by $\left(s-s_{2}\right)$, and putting $s=s_{2}$ we get

$\frac{\mathrm{V}}{\mathrm{L}} \frac{\mathrm{s}(\mathrm{s} \sin \theta+\omega \cos \theta)\left(\mathrm{s}-\mathrm{s}_{2}\right)}{(\mathrm{s}+\mathrm{j} \omega)(\mathrm{s}-\mathrm{j} \omega)\left(\mathrm{s}-\mathrm{s}_{2}\right)\left(\mathrm{s}-\mathrm{s}_{1}\right)}=\mathrm{k}_{2}+0$.

$\therefore \mathrm{k}_{2}=\frac{\mathrm{V}}{\mathrm{L}} \frac{\mathrm{s}_{2}\left(\mathrm{~s}_{2} \sin \theta+\omega \cos \theta\right)}{\left(\mathrm{s}_{2}+\mathrm{j} \omega\right)\left(\mathrm{s}_{2}-\mathrm{j} \omega\right)\left(\mathrm{s}_{2}-\mathrm{s}_{1}\right)}$

Multiplying eq (28) on both sides by $(s+j \omega)$, and putting $s=-j \omega$ we get

$\frac{\mathrm{V}}{\mathrm{L}} \frac{\mathrm{s}(\mathrm{s} \sin \theta+\omega \cos \theta)(\mathrm{s}+\mathrm{j} \omega)}{(\mathrm{s}+\mathrm{j} \omega)(\mathrm{s}-\mathrm{j} \omega)\left(\mathrm{s}-\mathrm{s}_{2}\right)\left(\mathrm{s}-\mathrm{s}_{1}\right)}=\mathrm{k}_{3}+0$,

$\therefore \mathrm{k}_{3}=\frac{\mathrm{V}}{\mathrm{L}} \frac{-\mathrm{j} \omega(-\mathrm{j} \omega \sin \theta+\omega \cos \theta)}{(-\mathrm{j} \omega-\mathrm{j} \omega)\left(-\mathrm{j} \omega-\mathrm{s}_{1}\right)\left(-\mathrm{j} \omega-\mathrm{s}_{2}\right)}$

$\mathrm{k}_{3}=\frac{\omega(\cos \theta-\mathrm{j} \sin \theta) \mathrm{V}}{2\left(\mathrm{~s}_{1}+\mathrm{j} \omega\right)\left(\mathrm{s}_{2}+\mathrm{j} \omega\right) \mathrm{L}}$

Multiplying eq (28) on both sides by $(s-j \omega)$, and putting $s=j \omega$ we get

$\frac{\mathrm{V}}{\mathrm{L}} \frac{\mathrm{s}(\mathrm{s} \sin \theta+\omega \cos \theta)(\mathrm{s}-\mathrm{j} \omega)}{(\mathrm{s}+\mathrm{j} \omega)(\mathrm{s}-\mathrm{j} \omega)\left(\mathrm{s}-\mathrm{s}_{2}\right)\left(\mathrm{s}-\mathrm{s}_{1}\right)}=\mathrm{k}_{4}+0$,

$\therefore \mathrm{k}_{4}=\frac{\mathrm{V}}{\mathrm{L}} \frac{-\mathrm{j} \omega(-\mathrm{j} \omega \sin \theta+\omega \cos \theta)}{(-\mathrm{j} \omega-\mathrm{j} \omega)\left(-\mathrm{j} \omega-\mathrm{s}_{1}\right)\left(-\mathrm{j} \omega-\mathrm{s}_{2}\right)}$

$\mathrm{k}_{4}=\frac{\omega(\cos \theta+\mathrm{j} \sin \theta) \mathrm{V}}{2\left(\mathrm{~s}_{1}-\mathrm{j} \omega\right)\left(\mathrm{s}_{2}-\mathrm{j} \omega\right) \mathrm{L}}$

Substituting the value of $\mathrm{k}_{1}, \mathrm{k}_{2}, \mathrm{k}_{3}$ and $\mathrm{k}_{4}$ in (28), and takinginverse laplace

transform, we get

$\mathrm{i}(\mathrm{t})=\mathrm{k}_{1} \mathrm{e}^{\mathrm{s}_{1} \mathrm{t}}+\mathrm{k}_{2} \mathrm{e}^{\mathrm{s}_{2} \mathrm{t}}+\mathrm{k}_{3} \mathrm{e}^{-\mathrm{j} \omega \mathrm{t}}+\mathrm{k}_{4} \mathrm{e}^{\mathrm{j} \omega \mathrm{t}}$

$\mathrm{i}(\mathrm{t})=\frac{\mathrm{v}}{\mathrm{L}}\left[\mathrm{k}_{1} \mathrm{e}^{\mathrm{s}_{1} \mathrm{t}}+\mathrm{k}_{2} \mathrm{e}^{\mathrm{s}_{2} \mathrm{t}}\right]+\frac{\mathrm{v}}{\mathrm{L}}\left[\mathrm{k}_{3} \mathrm{e}^{-\mathrm{j} \omega \mathrm{t}}+\mathrm{k}_{4} \mathrm{e}^{\mathrm{j} \omega \mathrm{t}}\right]$

Equation (36) consists of two parts. First part is made up of two roots $s_{1}$ and $s_{2}$, and second part consists of two transitory components due to $\mathrm{j} \omega \mathrm{t}$.

$\mathrm{i}(\mathrm{t})=\mathrm{i}(\mathrm{t})^{\prime}+\mathrm{i}(\mathrm{t})^{\prime \prime}$

$\mathrm{i}(\mathrm{t})^{\prime}=\frac{\mathrm{v}}{\mathrm{L}}\left[\mathrm{k}_{1} \mathrm{e}^{\mathrm{s}_{1} \mathrm{t}}+\mathrm{k}_{2} \mathrm{e}^{\mathrm{s}_{2} \mathrm{t}}\right]$,

substituting the value of $\mathrm{k}_{1}, \mathrm{k}_{2}$ we get

$\mathrm{i}(\mathrm{t})^{\prime}=\frac{\mathrm{v}}{\mathrm{L}}\left[\frac{\mathrm{s}_{1}\left(\mathrm{~s}_{1} \sin \theta+\omega \cos \theta\right)}{\left(\mathrm{s}_{1}+\mathrm{j} \omega\right)\left(\mathrm{s}_{1}-\mathrm{j} \omega\right)\left(\mathrm{s}_{1}-\mathrm{s}_{2}\right)} \mathrm{e}^{\mathrm{s}_{1} \mathrm{t}}+\frac{\mathrm{s}_{2}\left(\mathrm{~s}_{2} \sin \theta+\omega \cos \theta\right)}{\left(\mathrm{s}_{2}+\mathrm{j} \omega\right)\left(\mathrm{s}_{2}-\mathrm{j} \omega\right)\left(\mathrm{s}_{2}-\mathrm{s}_{1}\right)} \mathrm{e}^{\mathrm{s}_{2} \mathrm{t}}\right]$

$\mathrm{i}(\mathrm{t})^{\prime}=\frac{\mathrm{V}}{\mathrm{L}\left(\mathrm{s}_{1}-\mathrm{s}_{2}\right)}\left[\frac{\mathrm{s}_{1}\left(\mathrm{~s}_{1} \sin \theta+\omega \cos \theta\right)}{\left(\mathrm{s}_{1}+\mathrm{j} \omega\right)\left(\mathrm{s}_{1}-\mathrm{j} \omega\right)} \mathrm{e}^{\mathrm{s}_{1} \mathrm{t}}-\frac{\mathrm{s}_{2}\left(\mathrm{~s}_{2} \sin \theta+\omega \cos \theta\right)}{\left(\mathrm{s}_{2}+\mathrm{j} \omega\right)\left(\mathrm{s}_{2}-\mathrm{j} \omega\right)} \mathrm{e}^{\mathrm{s}_{2} \mathrm{t}}\right]$

Equation (39) can be written as

$\mathrm{i}(\mathrm{t})^{\prime}=\frac{\mathrm{V}}{\mathrm{L}\left(\mathrm{s}_{1}-\mathrm{s}_{2}\right)}\left[\mathrm{c}_{1} \mathrm{e}^{\mathrm{s}_{1} \mathrm{t}}-\mathrm{c}_{2} \mathrm{e}^{\mathrm{s}_{2} \mathrm{t}}\right]$

$\mathrm{Eq}(40)$ is a generalized case but if we consider,

Case $1 \mathrm{~s}_{1}=\mathrm{D}_{1}=\mathrm{k}_{1}+\mathrm{k}_{2}$ andD $\mathrm{D}_{2}=\mathrm{s}_{2}=\mathrm{k}_{1}-\mathrm{k}_{2}$

Case $2 \mathrm{~s}_{1}=\mathrm{D}_{1}=\mathrm{k}_{1}+\mathrm{jk}_{2}$ and $\mathrm{D}_{2}=\mathrm{s}_{2}=\mathrm{k}_{1}-\mathrm{jk}_{2}$

Case $3 \mathrm{~s}_{1}=\mathrm{D}_{1}=\mathrm{k}_{1}$ andD $\mathrm{D}_{2}=\mathrm{s}_{2}=\mathrm{k}_{1}$,

Then three different equations are possible.

Following is the derivation of second part,

$\mathrm{i}_{\mathrm{t}}^{\prime \prime}=\frac{\mathrm{V}}{\mathrm{L}}\left[\mathrm{k}_{3} \mathrm{e}^{-\mathrm{j} \omega \mathrm{t}}+\mathrm{k}_{4} \mathrm{e}^{\mathrm{j} \omega \mathrm{t}}\right]$

Substituting the value of $\mathrm{k}_{3}$ and $\mathrm{k}_{4}$, we get

$\mathrm{i}(\mathrm{t})^{\prime \prime}=\frac{\mathrm{v}}{2 \mathrm{~L}}\left[\frac{\omega(\cos \theta-\mathrm{j} \sin \theta) \mathrm{e}^{-\mathrm{j} \omega \mathrm{t}}}{\left(\mathrm{s}_{1}+\mathrm{j} \omega\right)\left(\mathrm{s}_{2}+\mathrm{j} \omega\right)}+\frac{\omega(\cos \theta+\mathrm{j} \sin \theta) \mathrm{e}^{\mathrm{j} \omega \mathrm{t}}}{\left(\mathrm{s}_{1}-\mathrm{j} \omega\right)\left(\mathrm{s}_{2}-\mathrm{j} \omega\right)}\right]$

$\mathrm{i}(\mathrm{t})^{\prime \prime}=\frac{\mathrm{V} \omega}{2 \mathrm{~L}}\left[\frac{\mathrm{e}^{-\mathrm{j} \theta} \mathrm{e}^{-\mathrm{j} \omega \mathrm{t}}}{\left(\mathrm{s}_{1}+\mathrm{j} \omega\right)\left(\mathrm{s}_{2}+\mathrm{j} \omega\right)}+\frac{\mathrm{e}^{\mathrm{j} \theta} \mathrm{e}^{\mathrm{j} \omega \mathrm{t}}}{\left(\mathrm{s}_{1}-\mathrm{j} \omega\right)\left(\mathrm{s}_{2}-\mathrm{j} \omega\right)}\right]$

$=\frac{V \omega}{2 L}\left[\frac{\left[\mathrm{e}^{-\mathrm{j}(\omega \mathrm{t}+\theta)}\left(\mathrm{s}_{1}-\mathrm{j} \omega\right)\left(\mathrm{s}_{2}-\mathrm{j} \omega\right)\right]+\mathrm{e}^{\mathrm{j}(\omega \mathrm{t}+\theta)}\left(\mathrm{s}_{1}+\mathrm{j} \omega\right)\left(\mathrm{s}_{2}+\mathrm{j} \omega\right)}{\left(\mathrm{s}_{1}{ }^{2}+\omega^{2}\right)\left(\mathrm{s}_{2}{ }^{2}+\omega^{2}\right)}\right]$

$=\frac{V \omega}{2 L} \frac{\left[\mathrm{e}^{-j(\omega t+\theta)}\left(s_{1} s_{2}-\omega^{2}-j \omega s_{1}-j \omega s_{2}\right)+e^{j(\omega t+\theta)}\left(s_{1} s_{2}-\omega^{2}+j \omega s_{1}+j \omega s_{2}\right)\right]}{\left(s_{1}^{2}+\omega^{2}\right)\left(s_{2}{ }^{2}+\omega^{2}\right)}$

$=\frac{V \omega}{2 L} \frac{\left[e^{-j(\omega t+\theta)}+e^{j(\omega t+\theta)}\left(s_{1} s_{2}-\omega^{2}\right)-j\left(\omega s_{1}+\omega s_{2}\right) e^{-j(\omega t+\theta)}-e^{j(\omega t+\theta)}\right]}{\left(s_{1}^{2}+\omega^{2}\right)\left(s_{2}{ }^{2}+\omega^{2}\right)}$

$=\frac{\mathrm{V} \omega}{\mathrm{L}} \frac{\left[\cos (\omega \mathrm{t}+\theta)\left(\frac{1}{\mathrm{LC}}-\omega^{2}\right)-\left(\frac{-\omega \mathrm{R}}{\mathrm{L}}\right) \sin (\omega \mathrm{t}+\theta)\right]}{\left(\mathrm{s}_{1}{ }^{2}+\omega^{2}\right)\left(\mathrm{s}_{2}{ }^{2}+\omega^{2}\right)}$

$=\frac{\mathrm{V} \omega}{\mathrm{L}} \frac{\left[\left(\frac{\omega \mathrm{R}}{\mathrm{L}}\right) \sin (\omega \mathrm{t}+\theta)-\cos (\omega \mathrm{t}+\theta)\left(\omega^{2}-\frac{1}{\mathrm{LC}}\right)\right]}{\left(\mathrm{s}_{1}{ }^{2}+\omega^{2}\right)\left(\mathrm{s}_{2}{ }^{2}+\omega^{2}\right)}$ 
Assuming

$\operatorname{Mcos} \varphi=\frac{\omega \mathrm{R}}{\mathrm{L}}$

And

$\operatorname{Msin} \varphi=\left(\omega^{2}-\frac{1}{\mathrm{LC}}\right)$

$\therefore \tan \varphi=\left(\frac{\omega \mathrm{L}-\frac{1}{\omega \mathrm{C}}}{\mathrm{R}}\right)$

or $\varphi=\tan ^{-1}\left(\frac{\omega \mathrm{L}-\frac{1}{\omega \mathrm{C}}}{\mathrm{R}}\right)$

$\mathrm{M}=\frac{\sqrt{\mathrm{R}^{2}+\left(\omega \mathrm{L}-\frac{1}{\omega \mathrm{C}}\right)^{2}}}{1}$

Equation (4) reduces to

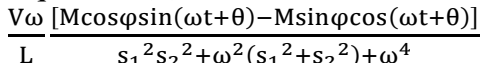

Substituting the value of $s_{1}{ }^{2} s_{2}{ }^{2}+\omega^{2}\left(s_{1}{ }^{2}+s_{2}{ }^{2}\right)+\omega^{4}$ in (54), we get

$=\frac{\mathrm{V} \omega[\mathrm{M} \sin (\omega \mathrm{t}+\theta-\varphi)]}{\mathrm{L}\left(\mathrm{s}_{1}{ }^{2} \mathrm{~s}_{2}{ }^{2}+\omega^{2}\left(\mathrm{~s}_{1}{ }^{2}+\mathrm{s}_{2}{ }^{2}\right)+\omega^{4}\right)}$

or

$\frac{\mathrm{V} \omega^{2} \mathrm{~L}^{2} \sqrt{\mathrm{R}^{2}+\left(\omega \mathrm{L}-\frac{1}{\omega \mathrm{C}}\right)^{2}}\left[\sin \left(\omega \mathrm{t}+\theta-\tan ^{-1}\left(\frac{\omega \mathrm{L}-\frac{1}{\omega \mathrm{C}}}{\mathrm{R}}\right)\right)\right]}{\mathrm{L}^{2}\left(\mathrm{R}^{2}+\left(\omega \mathrm{L}-\frac{1}{\omega \mathrm{C}}\right)^{2}\right) \omega^{2}}$

$\mathrm{i}(\mathrm{t})^{\prime \prime}=\frac{V \sin \left(\omega \mathrm{t}+\theta-\tan ^{-1}\left(\frac{\omega \mathrm{L}-\frac{1}{\omega \mathrm{C}}}{\mathrm{R}}\right)\right)}{\sqrt{\mathrm{R}^{2}+\left(\omega \mathrm{L}-\frac{1}{\omega \mathrm{C}}\right)^{2}}}$

$\mathrm{i}(\mathrm{t})=\mathrm{i}(\mathrm{t})^{\prime}+\mathrm{i}(\mathrm{t})^{\prime \prime}$

Substituting the value of $\mathrm{i}(\mathrm{t})^{\prime}$ andi( $(\mathrm{t})^{\prime \prime}$ from equation (39) and (57), we get

$i(t)=\frac{V}{L\left(s_{1}-s_{2}\right)}\left[\frac{s_{1}\left(s_{1} \sin \theta+\omega \cos \theta\right)}{\left(s_{1}+j \omega\right)\left(s_{1}-j \omega\right)} e^{s_{1} t}-\frac{s_{2}\left(s_{2} \sin \theta+\omega \cos \theta\right)}{\left(s_{2}+j \omega\right)\left(s_{2}-j \omega\right)} e^{s_{2} t}\right]+\frac{V \sin \left(\omega t+\theta-\tan ^{-1}\left(\frac{\omega L-\frac{1}{\omega C}}{R}\right)\right)}{\sqrt{R^{2}+\left(\omega L-\frac{1}{\omega C}\right)^{2}}}$

Generalizing the two equations without attempting to apply initial conditions at $\mathrm{t}=0$, for finding the value of $\mathrm{c}_{1}, \mathrm{c}_{2}, \mathrm{~s}_{1}$ and $\mathrm{s}_{1}$, similarity between the two equations derived by classical method and laplace transform method can be drawn in the following way.

Following is the generalized equation by Laplace Transform method.

Classical Method:

$\mathrm{i}(\mathrm{t})=\frac{\mathrm{v}}{\sqrt{\mathrm{R}^{2}+\left(\omega \mathrm{L}-\frac{1}{\omega \mathrm{C}}\right)^{2}}} \sin \left(\omega \mathrm{t}+\theta-\tan ^{-1}\left(\frac{\omega \mathrm{L}-\frac{1}{\omega \mathrm{C}}}{\mathrm{R}}\right)\right)+$

$\mathrm{c}_{1} \mathrm{e}^{\left(\mathrm{k}_{1}+\mathrm{k}_{2}\right) \mathrm{t}}-\mathrm{c}_{2} \mathrm{e}^{\left(\mathrm{k}_{1}-\mathrm{k}_{2}\right) \mathrm{t}}$

Laplace Transform Method:

$\mathrm{i}(\mathrm{t})=\frac{\mathrm{V} \sin \left(\omega \mathrm{t}+\theta-\tan ^{-1}\left(\frac{\omega \mathrm{L}-\frac{1}{\omega \mathrm{C}}}{\mathrm{R}}\right)\right)}{\sqrt{\mathrm{R}^{2}+\left(\omega \mathrm{L}-\frac{1}{\omega \mathrm{C}}\right)^{2}}}+$

$\left[\frac{\mathrm{V}}{\mathrm{L}} \frac{\mathrm{s}_{1}\left(\mathrm{~s}_{1} \sin \theta+\omega \cos \theta\right)}{\left(\mathrm{s}_{1}+\mathrm{j} \omega\right)\left(\mathrm{s}_{1}-\mathrm{j} \omega\right)\left(\mathrm{s}_{1}-\mathrm{s}_{2}\right)} \mathrm{e}^{\mathrm{s}_{1} \mathrm{t}}+\frac{\mathrm{V}}{\mathrm{L}} \frac{\mathrm{s}_{2}\left(\mathrm{~s}_{2} \sin \theta+\omega \cos \theta\right)}{\left(s_{2}+\mathrm{j} \omega\right)\left(\mathrm{s}_{2}-\mathrm{j} \omega\right)\left(\mathrm{s}_{2}-\mathrm{s}_{1}\right)} \mathrm{e}^{\mathrm{s}_{2} \mathrm{t}}\right]$

\subsubsection{Case 1 Above equations are same if we substitute the two roots $D 1=k 1+K 2=S 1$ and $D 2=k 1-k 2=S 2$}

And also

$\mathrm{c}_{1}=\frac{\mathrm{V}}{\mathrm{L}} \frac{\mathrm{s}_{1}\left(\mathrm{~s}_{1} \sin \theta+\omega \cos \theta\right)}{\left(\mathrm{s}_{1}+\mathrm{j} \omega\right)\left(\mathrm{s}_{1}-\mathrm{j} \omega\right)\left(\mathrm{s}_{1}-\mathrm{s}_{2}\right)}$

And

$c_{2}=-\frac{v}{L} \frac{s_{2}\left(s_{2} \sin \theta+\omega \cos \theta\right)}{\left(s_{2}+j \omega\right)\left(s_{2}-j \omega\right)\left(s_{2}-s_{1}\right)}$

Above table can be re written as for classical method by substituting as above

Classical Method

$\mathrm{i}(\mathrm{t})=\frac{\mathrm{V}}{\sqrt{\mathrm{R}^{2}+\left(\omega \mathrm{L}-\frac{1}{\omega \mathrm{C}}\right)^{2}}} \sin \left(\omega \mathrm{t}+\theta-\tan ^{-1}\left(\frac{\omega \mathrm{L}-\frac{1}{\omega \mathrm{C}}}{\mathrm{R}}\right)\right)+$

$\frac{\mathrm{V}}{\mathrm{L}} \frac{\mathrm{s}_{1}\left(\mathrm{~s}_{1} \sin \theta+\omega \cos \theta\right)}{\left(\mathrm{s}_{1}+\mathrm{j} \omega\right)\left(\mathrm{s}_{1}-\mathrm{j} \omega\right)\left(\mathrm{s}_{1}-\mathrm{s}_{2}\right)} \mathrm{e}^{\mathrm{s}_{1} \mathrm{t}}+\frac{\mathrm{V}}{\mathrm{L}} \frac{\mathrm{s}_{2}\left(\mathrm{~s}_{2} \sin \theta+\omega \cos \theta\right)}{\left(\mathrm{s}_{2}+\mathrm{j} \omega\right)\left(\mathrm{s}_{2}-\mathrm{j} \omega\right)\left(\mathrm{s}_{2}-\mathrm{s}_{1}\right)} \mathrm{e}^{\mathrm{s}_{2} \mathrm{t}}$ 
Laplace Transform Method:

$\mathrm{i}(\mathrm{t})=\frac{V \sin \left(\omega \mathrm{t}+\theta-\tan ^{-1}\left(\frac{\omega \mathrm{L}-\frac{1}{\omega \mathrm{C}}}{\mathrm{R}}\right)\right)}{\sqrt{\mathrm{R}^{2}+\left(\omega \mathrm{L}-\frac{1}{\omega \mathrm{C}}\right)^{2}}}+$

$\left[\frac{\mathrm{V}}{\mathrm{L}} \frac{\mathrm{s}_{1}\left(\mathrm{~s}_{1} \sin \theta+\omega \cos \theta\right)}{\left(\mathrm{s}_{1}+\mathrm{j} \omega\right)\left(\mathrm{s}_{1}-\mathrm{j} \omega\right)\left(\mathrm{s}_{1}-\mathrm{s}_{2}\right)} \mathrm{e}^{\mathrm{s}_{1} \mathrm{t}}+\frac{\mathrm{V}}{\mathrm{L}} \frac{\mathrm{s}_{2}\left(\mathrm{~s}_{2} \sin \theta+\omega \cos \theta\right)}{\left(\mathrm{s}_{2}+\mathrm{j} \omega\right)\left(\mathrm{s}_{2}-\mathrm{j} \omega\right)\left(\mathrm{s}_{2}-\mathrm{s}_{1}\right)} \mathrm{e}^{\mathrm{s}_{2} \mathrm{t}}\right]$

Above equations are same if we substitute the two roots $D_{1}=k_{1}+K_{2}=S_{1}$ and $D_{2}=k_{1}-k_{2}=S_{2}$

And also

$\mathrm{c}_{1}=\frac{\mathrm{v}}{\mathrm{L}} \frac{\mathrm{s}_{1}\left(\mathrm{~s}_{1} \sin \theta+\omega \cos \theta\right)}{\left(\mathrm{s}_{1}+\mathrm{j} \omega\right)\left(\mathrm{s}_{1}-\mathrm{j} \omega\right)\left(\mathrm{s}_{1}-\mathrm{s}_{2}\right)}$

And

$\mathrm{c}_{2}=-\frac{\mathrm{V}}{\mathrm{L}} \frac{\mathrm{s}_{2}\left(\mathrm{~s}_{2} \sin \theta+\omega \cos \theta\right)}{\left(\mathrm{s}_{2}+\mathrm{j} \omega\right)\left(\mathrm{s}_{2}-\mathrm{j} \omega\right)\left(\mathrm{s}_{2}-\mathrm{s}_{1}\right)}$

Above table can be re written as for Laplace Transform method by substituting as

$\mathrm{i}(\mathrm{t})=\frac{\mathrm{v}}{\sqrt{\mathrm{R}^{2}+\left(\omega \mathrm{L}-\frac{1}{\omega \mathrm{C}}\right)^{2}}} \sin \left(\omega \mathrm{t}+\theta-\tan ^{-1}\left(\frac{\omega \mathrm{L}-\frac{1}{\omega \mathrm{C}}}{\mathrm{R}}\right)\right)+\mathrm{c}_{1} \mathrm{e}^{\left(\mathrm{k}_{1}+\mathrm{k}_{2}\right) \mathrm{t}}-\mathrm{c}_{2} \mathrm{e}^{\left(\mathrm{k}_{1}-\mathrm{k}_{2}\right) \mathrm{t}}$

$\mathrm{i}(\mathrm{t})=\frac{\mathrm{V} \sin \left(\omega \mathrm{t}+\theta-\tan ^{-1}\left(\frac{\omega \mathrm{L}-\frac{1}{\omega \mathrm{C}}}{\mathrm{R}}\right)\right)}{\sqrt{\mathrm{R}^{2}+\left(\omega \mathrm{L}-\frac{1}{\omega \mathrm{C}}\right)^{2}}}+\left[\mathrm{c}_{1} \mathrm{e}^{\left(\mathrm{k}_{1}+\mathrm{k}_{2}\right) \mathrm{t}}-\mathrm{c}_{2} e^{\left(k_{1}-k_{2}\right) t}\right]$

Equatons shown are same.

1.2.2.2. Generalized equation case 2 , if we substitute the two roots $d 1=k 1+j k 2=s 1$ and $d 2=k 1-j k 2=s 2$ classical method

$i(t)=\frac{V}{\sqrt{R^{2}+\left(\omega L-\frac{1}{\omega C}\right)^{2}}} \sin \left(\omega t+\theta-\tan ^{-1}\left(\frac{\omega L-\frac{1}{\omega C}}{R}\right)\right)+e^{k_{1} t}\left[c_{1} \cos k_{2} t+c_{2} \sin k_{2} t\right]$
$i(t)=\frac{V \sin \left(\omega t+\theta-\tan ^{-1}\left(\frac{\omega L-\frac{1}{\omega C}}{R}\right)\right)}{\sqrt{R^{2}+\left(\omega L-\frac{1}{\omega C}\right)^{2}}}+$
$\left[\frac{V}{L} \frac{s_{1}\left(s_{1} \sin \theta+\omega \cos \theta\right)}{\left(s_{1}+j \omega\right)\left(s_{1}-j \omega\right)\left(s_{1}-s_{2}\right)} e^{s_{1} t}+\frac{V}{L} \frac{s_{2}\left(s_{2} \sin \theta+\omega \cos \theta\right)}{\left(s_{2}+j \omega\right)\left(s_{2}-j \omega\right)\left(s_{2}-s_{1}\right)} e^{s_{2} t}\right]$
$i(t)=\frac{V}{\sqrt{R^{2}+\left(\omega L-\frac{1}{\omega C}\right)^{2}}} \sin \left(\omega t+\theta-\tan ^{-1}\left(\frac{\omega L-\frac{1}{\omega C}}{R}\right)\right)+\mathrm{e}^{k_{1} t}\left[c_{1} \cos _{2} t+c_{2} \sin k_{2} t\right]$
$i(t)=\frac{V \sin \left(\omega t+\theta-\tan ^{-1}\left(\frac{\omega L-\frac{1}{\omega C}}{R}\right)\right)}{\sqrt{R^{2}+\left(\omega L-\frac{1}{\omega C}\right)^{2}}}+\frac{V\left[k_{1}{ }^{\prime} e^{s_{1} t}+k_{2}{ }^{\prime} e^{s_{2} t}\right]}{L\left(s_{1}-s_{2}\right)}$

If we substitute the two roots $D_{1}=k_{1}+j k_{2}=S_{1}$ and $D_{2}=k_{1}-j k_{2}=S_{2}$ in above equation, we get

$i(t)^{\prime}=\frac{V}{L(k 1+j k 2-(k 1-j k 2)}\left[k_{1}{ }^{\prime} e^{\left(k_{1}+j k_{2}\right) t}-k_{2}{ }^{\prime} e^{(k 1-j k 2) t}\right]$
$i(t)^{\prime}=\frac{V}{L(2 j) k_{2}}\left[\left(k_{1}{ }^{\prime} e^{k_{1} t} e^{j k_{2} t}\right)-\left(k_{2}{ }^{\prime} e^{k_{1} t} e^{-j k_{2} t}\right)\right]$

Rearranging and substituting value of $e^{j k_{2} t}$ and $e^{-j k_{2} t}$ in terms of cos and sin components

$i(t)^{\prime}=e^{k_{1} t}\left[\frac{V\left[\left(k_{1}{ }^{\prime}-k_{2}{ }^{\prime}\right) 2 \cos k_{2} t+\left(k_{1}{ }^{\prime}+k_{2}{ }^{\prime}\right) 2 \sin k_{2} t\right]}{L(2 j) k_{2}}\right]$

$i(t)^{\prime}=e^{k_{1} t}\left[c_{1} \cos k_{2} t+c_{2} \sin k_{2} t\right]$

where $c_{1}=\frac{V\left(k_{1}{ }^{\prime}-k_{2}{ }^{\prime}\right) 2}{2 j L k_{2}}$ and $c_{2}=\frac{V\left(k_{1}{ }^{\prime}+k_{2}{ }^{\prime}\right) 2}{2 j L k_{2}}$

which is same as equation by classical method if

we substitute

$i(t)=\frac{V}{\sqrt{R^{2}+\left(\omega L-\frac{1}{\omega C}\right)^{2}}} \sin \left(\omega t+\theta-\tan ^{-1}\left(\frac{\omega L-\frac{1}{\omega C}}{R}\right)\right)+e^{k_{1} t}\left[c_{1} \cos k_{2} t+c_{2} \sin k_{2} t\right]$

$i(t)=\frac{V \sin \left(\omega t+\theta-\tan ^{-1}\left(\frac{\omega L-\frac{1}{\omega C}}{R}\right)\right)}{\sqrt{R^{2}+\left(\omega L-\frac{1}{\omega C}\right)^{2}}}+\left[e^{k_{1} t}\left[c_{1} \cos k_{2} t+c_{2} \sin k_{2} t\right]\right]$

Equatons shown are same. 


\subsubsection{Generalised equation case 3 , if we substitute the two roots $d 1=k 1=s 1$ and $d 2=k 1=s 2$ classical $\operatorname{method}$}

$i(t)=\frac{V}{\sqrt{R^{2}+\left(\omega L-\frac{1}{\omega C}\right)^{2}}} \sin \left(\omega t+\theta-\tan ^{-1}\left(\frac{\omega L-\frac{1}{\omega C}}{R}\right)\right)+e^{k_{1} t}\left[c_{1}+c_{2}\right]$

Laplace transforms method:

$i(t)=\frac{V \sin \left(\omega t+\theta-\tan ^{-1}\left(\frac{\omega L-\frac{1}{\omega C}}{R}\right)\right)}{\sqrt{R^{2}+\left(\omega L-\frac{1}{\omega C}\right)^{2}}}+\left[\frac{V}{L} \frac{s_{1}\left(s_{1} \sin \theta+\omega \cos \theta\right)}{\left(s_{1}+j \omega\right)\left(s_{1}-j \omega\right)\left(s_{1}-s_{2}\right)} e^{s_{1} t}+\frac{V}{L} \frac{s_{2}\left(s_{2} \sin \theta+\omega \cos \theta\right)}{\left(s_{2}+j \omega\right)\left(s_{2}-j \omega\right)\left(s_{2}-s_{1}\right)} e^{s_{2} t}\right]$

In the above equation of $\mathrm{i}(\mathrm{t})$ by laplace transform method

$i(t)=\frac{V \sin \left(\omega t+\theta-\tan ^{-1}\left(\frac{\omega L-\frac{1}{\omega C}}{R}\right)\right)}{\sqrt{R^{2}+\left(\omega L-\frac{1}{\omega C}\right)^{2}}}+\left[\frac{V}{L} \frac{s_{1}\left(s_{1} \sin \theta+\omega \cos \theta\right)}{\left(s_{1}+j \omega\right)\left(s_{1}-j \omega\right)\left(s_{1}-s_{2}\right)} e^{s_{1} t}+\frac{V}{L} \frac{s_{2}\left(s_{2} \sin \theta+\omega \cos \theta\right)}{\left(s_{2}+j \omega\right)\left(s_{2}-j \omega\right)\left(s_{2}-s_{1}\right)} e^{s_{2} t}\right]$

Rearranging the second term with substitution of $\mathrm{D}_{1}=\mathrm{K}_{1}=\mathrm{S}_{1}=\mathrm{D}_{2}=\mathrm{K}_{1}=\mathrm{S}_{2}$ in the following equation

$\left[\frac{V}{L} \frac{s_{1}\left(s_{1} \sin \theta+\omega \cos \theta\right)}{\left(s_{1}+j \omega\right)\left(s_{1}-j \omega\right)\left(s_{1}-s_{2}\right)} e^{s_{1} t}+\frac{V}{L} \frac{s_{2}\left(s_{2} \sin \theta+\omega \cos \theta\right)}{\left(s_{2}+j \omega\right)\left(s_{2}-j \omega\right)\left(s_{2}-s_{1}\right)} e^{s_{2} t}\right]$

$\frac{V}{L} \frac{s_{1}\left(s_{1} \sin \theta+\omega \cos \theta\right)}{\left(s_{1}+j \omega\right)\left(s_{1}-j \omega\right)\left(s_{1}-s_{2}\right)} e^{k_{1} t}+\frac{V}{L} \frac{s_{2}\left(s_{2} \sin \theta+\omega \cos \theta\right)}{\left(s_{2}+j \omega\right)\left(s_{2}-j \omega\right)\left(s_{2}-s_{1}\right)} e^{k_{1} t}$

$\left[e^{k_{1} t}\left(\frac{V}{L} \frac{s_{1}\left(s_{1} \sin \theta+\omega \cos \theta\right)}{\left(s_{1}+j \omega\right)\left(s_{1}-j \omega\right)\left(s_{1}-s_{2}\right)}+\frac{V}{L} \frac{s_{2}\left(s_{2} \sin \theta+\omega \cos \theta\right)}{\left(s_{2}+j \omega\right)\left(s_{2}-j \omega\right)\left(s_{2}-s_{1}\right)}\right)\right]$

$=e^{k_{1} t}\left[c_{1}+c_{2}\right]$

$\left[\right.$ where $\left.c_{1}=\left(\frac{V}{L} \frac{s_{1}\left(s_{1} \sin \theta+\omega \cos \theta\right)}{\left(s_{1}+j \omega\right)\left(s_{1}-j \omega\right)\left(s_{1}-s_{2}\right)}\right)\right]$

And

$c_{2}=\left(\frac{V}{L} \frac{s_{2}\left(s_{2} \sin \theta+\omega \cos \theta\right)}{\left(s_{2}+j \omega\right)\left(s_{2}-j \omega\right)\left(s_{2}-s_{1}\right)}\right)$

With this substitution, Laplace Transform equation can be rewritten in following way,

$i(t)=\frac{V}{\sqrt{R^{2}+\left(\omega L-\frac{1}{\omega C}\right)^{2}}} \sin \left(\omega t+\theta-\tan ^{-1}\left(\frac{\omega L-\frac{1}{\omega C}}{R}\right)\right)+e^{k_{1} t}\left[c_{1}+c_{2}\right]$

$i(t)=\frac{V \sin \left(\omega t+\theta-\tan ^{-1}\left(\frac{\omega L-\frac{1}{\omega C}}{R}\right)\right)}{\sqrt{R^{2}+\left(\omega L-\frac{1}{\omega C}\right)^{2}}}+e^{k_{1} t}\left[c_{1}+c_{2}\right]$

Comparative for all the three different cases:

(i) $\mathrm{D}_{1}=\mathrm{k}_{1}+\mathrm{K}_{2}=\mathrm{S}_{1}$ and $\mathrm{D}_{2}=\mathrm{k}_{1}-\mathrm{k}_{2}=\mathrm{S}_{2}$

$$
\begin{aligned}
& i(t)=\frac{V}{\sqrt{R^{2}+\left(\omega L-\frac{1}{\omega C}\right)^{2}}} \sin \left(\omega t+\theta-\tan ^{-1}\left(\frac{\omega L-\frac{1}{\omega C}}{R}\right)\right)+c_{1} e^{\left(k_{1}+k_{2}\right) t}-c_{2} e^{\left(k_{1}-k_{2}\right) t} \\
& i(t)=\frac{V \sin \left(\omega t+\theta-\tan ^{-1}\left(\frac{\omega L-\frac{1}{\omega C}}{R}\right)\right)}{\sqrt{R^{2}+\left(\omega L-\frac{1}{\omega C}\right)^{2}}}+\left[c_{1} e^{\left(k_{1}+k_{2}\right) t}-c_{2} e^{\left(k_{1}-k_{2}\right) t}\right] \\
& i(t)=\frac{V}{\sqrt{R^{2}+\left(\omega L-\frac{1}{\omega C}\right)^{2}}} \sin \left(\omega t+\theta-\tan ^{-1}\left(\frac{\omega L-\frac{1}{\omega C}}{R}\right)\right)+ \\
& \frac{V}{L} \frac{s_{1}\left(s_{1} \sin \theta+\omega \cos \theta\right)}{\left(s_{1}+j \omega\right)\left(s_{1}-j \omega\right)\left(s_{1}-s_{2}\right)} e^{s_{1} t}+\frac{V}{L} \frac{s_{2}\left(s_{2} \sin \theta+\omega \cos \theta\right)}{\left(s_{2}+j \omega\right)\left(s_{2}-j \omega\right)\left(s_{2}-s_{1}\right)} e^{s_{2} t} \\
& i(t)=\frac{V \sin \left(\omega t+\theta-\tan -1\left(\frac{\omega L-\frac{1}{\omega C}}{R}\right)\right)}{\sqrt{R^{2}+\left(\omega L-\frac{1}{\omega C}\right)^{2}}}+ \\
& {\left[\frac{V}{L} \frac{s_{1}\left(s_{1} \sin \theta+\omega \cos \theta\right)}{\left(s_{1}+j \omega\right)\left(s_{1}-j \omega\right)\left(s_{1}-s_{2}\right)} e^{s_{1} t}+\frac{V}{L} \frac{s_{2}\left(s_{2} \sin \theta+\omega \cos \theta\right)}{\left(s_{2}+j \omega\right)\left(s_{2}-j \omega\right)\left(s_{2}-s_{1}\right)} e^{s_{2} t}\right]}
\end{aligned}
$$

(ii) $\mathrm{D}_{1}=\mathrm{k}_{1}+\mathrm{j} \mathrm{k}_{2}=\mathrm{S}_{1}$ and $\mathrm{D}_{2}=\mathrm{k}_{1}-\mathrm{j} \mathrm{k}_{2}=\mathrm{S}_{2}$

$i(t)=\frac{V}{\sqrt{R^{2}+\left(\omega L-\frac{1}{\omega C}\right)^{2}}} \sin \left(\omega t+\theta-\tan ^{-1}\left(\frac{\omega L-\frac{1}{\omega C}}{R}\right)\right)+e^{k_{1} t}\left[c_{1} \cos k_{2} t+c_{2} \sin k_{2} t\right]$

$i(t)=\frac{V \sin \left(\omega t+\theta-\tan ^{-1}\left(\frac{\omega L-\frac{1}{\omega C}}{R}\right)\right)}{\sqrt{R^{2}+\left(\omega L-\frac{1}{\omega C}\right)^{2}}}+\left[e^{k_{1} t}\left[c_{1} \cos _{2} t+c_{2} \sin _{2} t\right]\right]$

(iii) $\mathrm{D}_{1}=\mathrm{K}_{1}=\mathrm{S}_{1}=\mathrm{D}_{2}=\mathrm{K}_{1}=\mathrm{S}_{2}$

$i(t)=\frac{V}{\sqrt{R^{2}+\left(\omega L-\frac{1}{\omega C}\right)^{2}}} \sin \left(\omega t+\theta-\tan ^{-1}\left(\frac{\omega L-\frac{1}{\omega C}}{R}\right)\right)+e^{k_{1} t}\left[c_{1}+c_{2}\right]$ 
$\mathrm{i}(\mathrm{t})=\frac{\operatorname{vsin}\left(\omega \mathrm{t}+\theta-\tan ^{-1}\left(\frac{\omega \mathrm{L}-\frac{1}{\omega \mathrm{C}}}{\mathrm{C}}\right)\right)}{\sqrt{\mathrm{R}^{2}+\left(\omega \mathrm{L}-\frac{1}{\omega \mathrm{C}}\right)^{2}}}+\mathrm{e}^{\mathrm{k}_{1} \mathrm{t}}\left[\mathrm{c}_{1}+\mathrm{c}_{2}\right]$

\section{Conclusion}

It is proved that equations governing sinusoidal excitation to R-L-C series circuit for transient behavior with storage elements initially in uncharged state are second order differential equations of non-homogeneous type. The mathematical methods of engineering namely classical method and Laplace Transform method have given similar results for the analysis which was carried out in this work. Also cases of over damped, under damped and critically damped condition was evaluated significant for natural response of R-L-C AC series circuit. Laplace transform method is proved to be a better tool with respect to initialization condition in comparison to classical method. Thus analysis of R-L-C AC series circuit with sinusoidal excitation during transient condition can be utilized to explain the phenomena occurring on transmission line.

\section{Acknowledgement}

I am thankful to department of Electrical Engineering for giving me an opportunity to teach a course on Basic Electrical Technology under which the topic is covered.

\section{References}

[1] A Sudhakar \& Shyammohan S Palli. (2010)." Circuits and Networks, Analysis and Synthesis, Fourth Edition, McGraw-Hill Education India Pvt.Ltd. Upper Saddle River: Prentice hall.

[2] B.L.Theraja \& A.K.Theraja,"A Textbook of Electrical Technology, S Chand \& Company Ltd, (2008). 